\section{Adolescentes e uso de preservativos: as escolhas dos jovens de três capitais brasileiras na iniciação e na última relação sexual}

\author{
Teenagers and condom use: \\ choices by young Brazilians from three Brazilian \\ State capitals in their first and last sexual intercourse
}

\author{
1 Departamento de Medicina \\ Social, Universidade Federal \\ de Pelotas, Pelotas, Brasil. \\ 2 Programa de Pós-graduação \\ em Epidemiologia \\ Universidade Federal \\ do Rio Grande do Sul, \\ Porto Alegre, Brasil. \\ 3 Núcleo de Pesquisa em \\ Antropologia do Corpo e da \\ Saúde, Porto Alegre, Brasil. \\ Correspondência \\ A. M. F. B. Teixeira \\ Departamento de Medicina \\ Social, Faculdade \\ de Medicina, Universidade \\ Federal de Pelotas. \\ Av. Duque de Caxias 250, \\ Pelotas, RS 96001-970, Brasil. \\ ateixeira@epidemio-ufpel.org.br
}

\begin{abstract}
Condom use has increased among Brazilian youth, although condoms are not used in all sexual relations; in addition, their use varies over the course of an individual's affective and sexual history. This study focused on the prevalence and factors associated with condom use during sexual initiation and in the most recent sexual relations in young men and women (1824 years of age). The data are from the GRAVAD Research Project, a cross-sectional study with a stratified probabilistic sample using household interviews in three Brazilian State capitals. The analysis used multinomial logistic regression with a hierarchical model. Prevalence of condom use in first sexual intercourse among individuals who used any contraceptive method was $80.7 \%$ for females and $88.6 \%$ for males. This proportion dropped to $38.8 \%$ and $56 \%$, respectively, for the most recent intercourse. In both events, and for both genders, condom use was associated with social belonging and age at initiation. Condom use at sexual initiation was correlated with use in the most recent intercourse $(O R=2.42$ for males and 1.89 for females). Increased condom use among youth does not mean continuing use. Women used condoms less than men in the events studied here.
\end{abstract}

Adolescent; Sexuality; Condoms; Gender Identity
Ana Maria Ferreira Borges Teixeira 1,2 Daniela Riva Knauth 2,3

Jandyra Maria Guimarães Fachel 2 Andrea Fachel Leal 3

\section{Introdução}

O número de jovens de 16 a 24 anos, no Brasil, representa $18 \%$ da população total do país, segundo dados do Instituto Brasileiro de Geografia e Estatística (IBGE) em 2002. Apesar do número elevado, muitos desses jovens ainda não têm acesso a informações e serviços adequados ao atendimento de suas necessidades em termos de saúde sexual e reprodutiva que os estimulem a tomar decisões de maneira livre e responsável 1.

As pesquisas sobre saúde sexual no Brasil, desenvolvidas pela BEMFAM (Bem-Estar Familiar no Brasil) em 1986 e 1996, vêm apontando para um aumento relativo da gravidez abaixo dos vinte anos, proporcionalmente à diminuição em todas as demais faixas etárias. Tendência que também ocorre em outros países, tanto naqueles em desenvolvimento, como nos desenvolvidos, tais como Inglaterra e Estados Unidos 2,3.

A literatura sobre o tema destaca as complicações biológicas e emocionais da gravidez na adolescência e as infecções sexualmente transmissíveis dentre os desfechos desfavoráveis 3,4 . Os aspectos sociais, como abandono escolar, limitação das oportunidades ocupacionais e de rendimentos futuros, são também ressaltados 1,4 .

A epidemia da AIDS e a gravidez deram visibilidade à sexualidade juvenil. As diferentes formas de intervenção direcionadas para o pú- 
blico jovem resultaram na difusão e no aumento do uso de preservativos entre essa parte da população 2,3,5,6,7. Contudo, se o uso de preservativo aumentou entre os jovens, ele ainda não é utilizado por todos e nem em todas as relações sexuais. Dentre os muitos estudos que têm sido realizados tendo como objeto os adolescentes e o uso de métodos de proteção/contracepção, vários demonstram que o conhecimento sobre os métodos anticoncepcionais existentes é elevado, o que não implica necessariamente o uso adequado ou regular destes e, apesar do aumento considerável desse uso nos últimos anos, ainda deixa a desejar 1,2,3,5,7,8.

É sabido que inúmeros fatores estão associados ao uso de métodos anticoncepcionais e/ou de proteção pessoal durante os relacionamentos afetivo-sexuais. Entre eles podemos citar o grau de conhecimento sobre as questões reprodutivas, sobre a atuação dos contraceptivos, especificidades relacionadas ao gênero, o tipo de envolvimento afetivo do momento, questões financeiras e de acesso aos métodos, bem como o grau de liberdade e de autonomia alcançados nessa faixa etária.

Buscando, assim, contribuir para a ampliação do conhecimento sobre a sexualidade juvenil brasileira, este estudo tem por objetivo identificar quais os fatores relacionados ao uso do preservativo pelos jovens. Para tanto, analisaremos a declaração de uso do preservativo em dois eventos da trajetória dos jovens, a primeira e a última relação sexual.

\section{Metodologia}

Os dados analisados neste trabalho fazem parte do material coletado pela pesquisa Gravidez na Adolescência: Estudo Multicêntrico sobre Jovens, Sexualidade e Reprodução no Brasil, denominada Pesquisa GRAVAD, que é uma investigação multicêntrica sobre sexualidade e gravidez na juventude. Foi realizado um estudo transversal, com amostragem probabilística estratificada, por setores censitários, representativa dos jovens de 18 anos a 24 anos, de ambos os sexos, nas cidades de Porto Alegre, Rio de Janeiro e Salvador, Brasil, no ano de 2002. Evitouse trabalhar com a faixa etária definida habitualmente como adolescência, dos 10 aos 19 anos, a fim de reconstruir as trajetórias afetivas e sexuais dos jovens e assegurar um certo distanciamento que possibilitasse a avaliação retrospectiva das experiências vividas e suas conseqüências, obtendo-se, ao mesmo tempo, uma proximidade temporal que permitisse a rememoração de eventos de modo mais acurado 9 .
Para a coleta dos dados, foi utilizado um questionário padronizado, com perguntas fechadas, aplicadas individualmente no domicílio por entrevistadores treinados. A amostra foi aleatória, selecionada em três estágios. No primeiro, foi selecionada uma amostra estratificada de setores censitários; no segundo, uma amostra de domicílios, e, no terceiro, foi selecionada a pessoa para ser entrevistada, obedecendo a critérios de restrições definidos.

Os setores censitários foram classificados em cinco estratos, conforme situação sócioeconômica dos habitantes. Os setores favelados (sub-normais pelo IBGE), formaram o estrato "zero". Os demais setores foram alocados em quatro estratos, baseados na renda média do chefe do domicílio e proporção de chefes com 12 ou mais anos de escolaridade.

Como o tamanho da amostra foi inicialmente calculado baseado nos dados existentes sobre número de partos na adolescência, para validar a presente análise foi realizado um cálculo de amostra, a partir de dados sobre uso de preservativos. A prevalência de uso de preservativos entre os jovens foi considerada de $55 \%$ 7,8 . Para o cálculo da amostra visando a conhecer a prevalência dos fatores associados ao uso de preservativo, testou-se cada uma das variáveis explicativas de interesse. Para um poder de $80 \%$ e nível de confiança de $95 \%$, a amostra de jovens que foi entrevistada tinha condições de detectar uma razão de risco de 1,2 ou mais, já considerando uma taxa de perda de $20 \%$.

Para avaliar a prevalência do uso de preservativos, o estudo analisou dois desfechos. $\mathrm{O}$ primeiro refere-se ao uso de preservativo na iniciação sexual e o segundo, ao seu uso na última relação sexual (a que ocorreu mais recentemente). Para a construção dos desfechos, uso de preservativo significa o uso do método isoladamente ou em associação com algum outro método. O desfecho foi construído com três categorias: "usou preservativo", "usou outro tipo de método" e "não usou nenhum método". Decidiu-se não agregar as duas últimas categorias por serem conceitualmente diferentes. Os jovens que utilizaram outros tipos de métodos que não o preservativo não podem ser igualados aos que não tomaram nenhum cuidado.

Para a análise estatística, utilizou-se o programa Stata versão 8.0 (Stata Corporation, College Station, Estados Unidos), considerando a complexidade do desenho amostral. A amostra foi ponderada com base nas probabilidades com que foram selecionadas as unidades de cada estágio correspondente. Na análise bruta, utilizou-se o teste qui-quadrado e regressão logística multinomial. Na análise ajustada, para 
o controle dos possíveis fatores de confusão, foi utilizada a regressão logística multinomial, sendo as variáveis introduzidas na regressão seguindo um modelo hierarquizado, conforme os níveis propostos num modelo conceitual 10. Essa análise avalia o efeito de cada variável explicativa, controlando para outras variáveis do mesmo nível e de níveis superiores. Foram mantidas no modelo de regressão todas as variáveis que apresentaram valor $\mathrm{p}<0,2011$. A permanência ou não das variáveis no modelo está vinculada exclusivamente ao comportamento destas em relação à categoria do desfecho "usou preservativo", considerando-se a categoria "não usou nenhum método" como a categoria de referência.

No modelo hierarquizado proposto, as variáveis estudadas com o uso de preservativo na primeira relação sexual foram: sexo, idade no dia da entrevista, cor da pele do jovem e escolaridade materna (nível mais distal); escolaridade do adolescente e religião que segue (segundo nível); se as primeiras informações sobre métodos anticoncepcionais foram dadas pela mãe e/ou pelo pai; idade que tinha na iniciação sexual, quem foi o parceiro, diferença etária entre parceiros e se conversaram sobre métodos antes da iniciação sexual (terceiro nível). Quanto ao segundo desfecho, uso de preservativo na última relação, além das variáveis já citadas no primeiro desfecho, mantidas nos mesmos níveis, foram avaliados também o uso de preservativo na iniciação sexual (quarto nível); quem era o parceiro da última relação sexual, tempo de vida sexual, número total de parceiros na vida e os tipos de trajetórias afetivas (quinto nível).

Excluíram-se desta análise os jovens que se declararam virgens (587), os que referiram relações com pessoas do mesmo sexo (20) e aqueles que não informaram se já haviam tido relações sexuais (8), pois não respondiam às questões referentes ao uso de métodos. Quanto à última relação sexual somente foi avaliada a ocorrida nos últimos 12 meses.

\section{Resultados}

Dos 4.634 jovens entrevistados, 4.039 (87,18\%) relataram já ter tido relações sexuais alguma vez na vida. Destes, $93,5 \%$ eram rapazes e $81,8 \%$ moças. Declararam-se virgens 587 (12,67\%). Para este estudo, foram considerados os 4.019 jovens analisados após as exclusões efetuadas. Para as análises referentes à última relação foram estudados 3.836 jovens.

As características sócio-econômicas e demográficas da amostra encontram-se descritas na Tabela 1. Nas Tabelas 2 e 3 encontram-se as características biográficas na primeira e última relação sexual. Ambos os pais forneceram aos jovens as primeiras orientações sobre métodos anticoncepcionais, as mães superando os pais como a fonte das primeiras informações e num percentual um pouco mais elevado para as moças do que para os rapazes. Já os rapazes foram informados pelo pai num percentual de $27,1 \%$, enquanto para as moças este percentual é de apenas 7,3\% (Tabela 2).

Existe diferença quanto aos tipos de relacionamentos na trajetória afetiva. Os rapazes declararam nenhum relacionamento estável em $9,4 \%$ das respostas, enquanto as moças, em apenas $2,2 \%$. Para estas predomina apenas um relacionamento estável, mas mais da metade, de ambos os sexos, referiu ter tido mais de um relacionamento estável (Tabela 3 ).

Na primeira relação sexual, o parceiro foi predominantemente o namorado, companheiro ou esposo para as moças e a pessoa com quem "ficou" ou garota de programa para os rapazes. Nenhuma moça referiu iniciação com profissional do sexo. Quanto à diferença etária entre os parceiros nessa relação, para as moças foram mais freqüentes homens mais velhos ou muito mais velhos e para os rapazes, parceiras da mesma idade ou mais jovens (Tabela 2). Já na última relação sexual, comparando com a primeira relação, a maioria das moças $(79,6 \%)$ e dos rapazes $(60,5 \%)$ teve como parceiro o atual. Chama a atenção que os rapazes referiram uma parceira eventual mais freqüentemente que as moças nesta ocasião (Tabela 3).

Quanto a conversarem sobre métodos anticoncepcionais com o parceiro, antes da primeira relação, as moças o fizeram mais do que os rapazes com suas parceiras (Tabela 2 ).

Entre todos os 4.019 jovens que já tiveram alguma relação sexual, $60 \%$ das moças e $63,8 \%$ dos rapazes usaram preservativo na primeira relação. Ao se analisarem somente os que referiram a utilização de algum tipo de método nessa relação (independentemente do tipo), o preservativo foi o mais citado, sendo este o método usado por $80,7 \%$ das moças e $88,6 \%$ dos rapazes, seguido pelo anticoncepcional oral (Tabela 2). Quando se avalia a última relação sexual, o uso de preservativo cai para $38,8 \%$ e $56 \%$, para moças e rapazes, respectivamente. Já o anticoncepcional oral aparece como o método mais freqüente (Tabela 3 ).

Para os jovens que declararam não ter usado nenhum método, foi perguntado o motivo do não uso. Na primeira relação sexual, dentre os motivos alegados pelas moças e rapazes respectivamente, foram citados: "nem pensaram 
Características sócio-econômicas e demográficas dos jovens de 18 a 24 anos, em Porto Alegre, Rio de Janeiro e Salvador, Brasil, 2002 ( $N=4.019$; dados ponderados).

\begin{tabular}{|c|c|c|c|c|}
\hline \multirow[t]{2}{*}{ Variáveis } & \multicolumn{2}{|c|}{ Feminino } & \multicolumn{2}{|c|}{ Masculino } \\
\hline & Freqüência & $\%$ & Freqüência & $\%$ \\
\hline \multicolumn{5}{|l|}{ Cor } \\
\hline Branca & 977 & 48,6 & 1.030 & 46,3 \\
\hline Negra/Parda & 851 & 51,4 & 843 & 53,7 \\
\hline \multicolumn{5}{|c|}{ Idade na entrevista (anos) } \\
\hline Até 19 & 479 & 24,7 & 576 & 26,2 \\
\hline$>19$ & 1.509 & 75,3 & 1.455 & 73,8 \\
\hline \multicolumn{5}{|c|}{ Escolaridade do jovem (anos) } \\
\hline Até 7 & 408 & 21,3 & 477 & 26,5 \\
\hline $8-10$ & 474 & 27,2 & 516 & 28,3 \\
\hline 11 ou + & 1.078 & 51,6 & 1.008 & 45,1 \\
\hline \multicolumn{5}{|l|}{ Segue religião } \\
\hline Sim & 1.205 & 65,6 & 931 & 49,1 \\
\hline Não & 770 & 34,4 & 1.097 & 50,9 \\
\hline \multicolumn{5}{|c|}{ Escolaridade materna (anos) } \\
\hline Nenhuma & 117 & 7,8 & 83 & 4,9 \\
\hline Até 7 & 661 & 35,8 & 605 & 37,2 \\
\hline $8-10$ & 317 & 18,7 & 319 & 17,4 \\
\hline 11 ou + & 795 & 37,6 & 893 & 40,6 \\
\hline
\end{tabular}

Os totais não coincidem em virtude da falta de informações para algumas variáveis.

nisso”, 69,6\% e 73,9\%; “pensava que não podia engravidar", 13,3\% e 9,6\%, "não sabiam como obter métodos", por $3 \%$ e 6,6\%, e "era responsabilidade do parceiro", 1,5\% e 3,7\%. Já na última relação sexual, embora os três primeiros motivos permanecessem como os mais mencionados, observa-se uma queda no primeiro $(41,9 \%$ e $39,4 \%$ ) e o aparecimento de referências ao desejo de ter filhos, seja do próprio entrevistado ou de seu parceiro.

A comparação dos dados da análise ajustada da iniciação e da última relação sexual (Tabelas 4 e 5) aponta que as variáveis que se mantêm associadas, para ambos os sexos, no que concerne ao uso do preservativo são o pertencimento social (cuja proxy utilizada aqui é a variável escolaridade da mãe) e a idade da iniciação sexual. A variável escolaridade da mãe, entre os rapazes, apesar de não mostrar associação com o uso de preservativo em cada uma de suas categorias, apresenta um aumento dos odds ratio diretamente proporcional ao aumento da escolaridade da mãe, e o teste de Wald para tendência apresentou associação significativa ( $p=0,039$ ). Assim, o uso continuado do preservativo se verifica entre os jovens cuja mãe possui uma escolaridade mais elevada $\mathrm{e}$ que iniciaram a vida sexual mais tardiamente. A comparação entre os dois eventos aponta também para o fato de que o uso de preservativo na iniciação apresenta forte associação com o uso na última relação. Os rapazes que usaram preservativo na primeira relação apresentaram um OR = 2,42 (IC95\%: 1,48-3,95) de uso na última relação comparando-se com os que não utilizaram nenhum método. Já as moças apresentaram OR = 1,89 (IC95\%: 1,16-3,09).

Ainda na análise ajustada da última relação sexual, as variáveis relativas à classificação do parceiro e ao número de parceiros sexuais que teve na trajetória também apresentaram associação para ambos os sexos. No universo masculino, o uso do preservativo com um parceiro classificado como eventual foi significativo, $\mathrm{OR}=$ 3,62 (IC95\%: 1,60-8,16), quando comparado com o parceiro com o qual o entrevistado está se relacionando sexual e afetivamente, isto é, com o parceiro considerado estável. Entre as mulheres, o uso do preservativo com um ex-parceiro (ex-marido ou ex-namorado) apresentou 


\section{Tabela 2}

Características biográficas da primeira relação sexual dos jovens de 18 a 24 anos, em Porto Alegre, Rio de Janeiro e Salvador, Brasil, 2002 ( $N=4.019$; dados ponderados).

\begin{tabular}{|c|c|c|c|c|}
\hline \multirow{2}{*}{ Variáveis } & \multicolumn{2}{|c|}{ Feminino } & \multicolumn{2}{|c|}{ Masculino } \\
\hline & Freqüência & $\%$ & Freqüência & $\%$ \\
\hline \multicolumn{5}{|l|}{ Primeiras informações sobre contracepção com mãe } \\
\hline Sim & 920 & 44,2 & 817 & 39,6 \\
\hline Não & 1.066 & 55,8 & 1.204 & 60,4 \\
\hline \multicolumn{5}{|l|}{ Primeiras informações sobre contracepção com pai } \\
\hline $\operatorname{Sim}$ & 157 & 7,3 & 572 & 27,1 \\
\hline Não & 1.829 & 92,7 & 1.449 & 72,9 \\
\hline \multicolumn{5}{|l|}{ Idade na ocasião } \\
\hline Precoce (rapazes: até 14 anos; moças: até 15 anos) & 585 & 28,2 & 584 & 29,6 \\
\hline Média (rapazes: 15-16 anos; moças: 16-17 anos) & 709 & 35,3 & 847 & 40,6 \\
\hline Tardia (rapazes: 17-24 anos; moças: 18-24 anos) & 687 & 36,4 & 591 & 29,8 \\
\hline \multicolumn{5}{|l|}{ Parceria } \\
\hline Pessoa com quem ficou/Garoto(a) de programa & 200 & 8,9 & 1.062 & 53,1 \\
\hline Namorado(a)/Marido/Esposa/Companheiro(a) & 1.766 & 91,1 & 915 & 46,9 \\
\hline \multicolumn{5}{|l|}{ Diferença etária entre parceria } \\
\hline Parceiro(a) mais jovem (2 anos e + ) & 28 & 1,9 & 187 & 9,7 \\
\hline Mesma idade (1 ano a - ou + ) & 458 & 23,9 & 988 & 51,8 \\
\hline Parceiro(a) mais velho $(2-4$ anos a +$)$ & 761 & 36,2 & 430 & 23,7 \\
\hline Parceiro(a) muito mais velho ( 5 anos e + ) & 712 & 38,0 & 279 & 14,9 \\
\hline \multicolumn{5}{|l|}{ Conversaram sobre métodos antes da relação } \\
\hline Sim & 1.208 & 62,0 & 810 & 40,7 \\
\hline Não & 763 & 38,0 & 1.197 & 59,3 \\
\hline \multicolumn{5}{|l|}{ Utilizaram proteção/Contracepção } \\
\hline $\operatorname{Sim}$ & 1.405 & 69,8 & 1.433 & 68,2 \\
\hline Não & 564 & 30,2 & 575 & 31,8 \\
\hline \multicolumn{5}{|l|}{$\begin{array}{l}\text { Métodos utilizados pelos } 2.838 \text { que fizeram } \\
\text { uso de contracepção/Proteção }\end{array}$} \\
\hline Preservativo & 1.155 & 80,7 & 1.312 & 88,6 \\
\hline Anticoncepcional oral & 189 & 14,9 & 87 & 8,3 \\
\hline Coito interrompido & 23 & 2,6 & 18 & 1,7 \\
\hline Injeção & 10 & 0,7 & 6 & 0,5 \\
\hline Tabela & 4 & 0,3 & 6 & 0,4 \\
\hline DIU & - & - & 3 & 0,1 \\
\hline Mais de um método & 11 & 0,3 & 6 & 0,2 \\
\hline Outros métodos & 6 & 0,4 & 2 & 0,2 \\
\hline
\end{tabular}

Os totais não coincidem em virtude da falta de informações para algumas variáveis. 
Características biográficas da última relação sexual dos jovens de 18 a 24 anos, em Porto Alegre, Rio de Janeiro e Salvador, Brasil, 2002 ( $N$ = 3.836; dados ponderados).

\begin{tabular}{|c|c|c|c|c|}
\hline \multirow[t]{2}{*}{ Variáveis } & \multicolumn{2}{|c|}{ Feminino } & \multicolumn{2}{|c|}{ Masculino } \\
\hline & Freqüência & $\%$ & Freqüência & $\%$ \\
\hline \multicolumn{5}{|l|}{ Parceria } \\
\hline Parceiro(a) atual & 1.485 & 79,6 & 1.148 & 60,5 \\
\hline Ex-marido/Ex-esposa/Ex-namorado(a) & 259 & 14,4 & 211 & 11,8 \\
\hline Parceiro eventual & 134 & 6,0 & 557 & 27,7 \\
\hline \multicolumn{5}{|c|}{ Tempo de vida sexualmente ativa (em anos) } \\
\hline Até 3 & 755 & 40,5 & 487 & 24,1 \\
\hline $4-6$ & 755 & 42,6 & 795 & 41,0 \\
\hline 7 ou + & 383 & 16,9 & 650 & 34,9 \\
\hline \multicolumn{5}{|l|}{ Número de parceiros sexuais na vida } \\
\hline 1 & 669 & 35,5 & 102 & 6,3 \\
\hline $2-4$ & 881 & 47,7 & 417 & 23,2 \\
\hline 5 ou + & 318 & 16,8 & 1.155 & 70,4 \\
\hline \multicolumn{5}{|l|}{ Tipos de trajetórias sexuais } \\
\hline Nenhum relacionamento estável & 37 & 2,2 & 204 & 9,4 \\
\hline Um relacionamento estável & 891 & 46,2 & 561 & 28,6 \\
\hline Mais de um relacionamento estável & 968 & 51,6 & 1.169 & 62,1 \\
\hline \multicolumn{5}{|l|}{ Usou preservativo } \\
\hline Sim & 773 & 38,8 & 1.136 & 56,0 \\
\hline Não & 1.089 & 61,2 & 774 & 44,0 \\
\hline \multicolumn{5}{|l|}{ Usou outro método que não camisinha } \\
\hline Sim & 1.123 & 57,3 & 789 & 39,3 \\
\hline Não & 745 & 42,7 & 1.097 & 60,7 \\
\hline \multicolumn{5}{|c|}{ Principal método usado nessa última relação sexual } \\
\hline Anticoncepcional oral & 891 & 79,1 & 659 & 83,5 \\
\hline Injeção/Implante & 94 & 8,0 & 36 & 4,2 \\
\hline DIU & 40 & 4,2 & 19 & 1,9 \\
\hline Diafragma & - & - & 1 & 0,1 \\
\hline Coito interrompido & 35 & 4,6 & 34 & 5,1 \\
\hline Tabela & 12 & 1,2 & 8 & 1,6 \\
\hline Mais de um método & 25 & 0,9 & 20 & 2,2 \\
\hline Outro & 28 & 2,0 & 11 & 1,4 \\
\hline
\end{tabular}

Os totais não coincidem em virtude da falta de informações para algumas variáveis.

OR = 2,0 (IC95\%: 1,06-3,82) e com um parceiro tido como eventual, apresentou $\mathrm{OR}=16,38$ (IC95\%: 4,05-66,19), em relação ao parceiro estável. O intervalo de confiança deste último foi amplo, provavelmente em virtude do pequeno número de moças que se relacionaram com parceiro eventual.

A relação entre uso do preservativo na última relação com o número de parceiros sexuais na vida apresentou associação inversa entre ra- pazes e moças. Para os rapazes, o uso de preservativo foi maior quando comparados dois a quatro parceiros com um único $(\mathrm{OR}=3,77$; IC95\%: 1,23-11,51), enquanto, para as moças, houve uma tendência de diminuição do uso com o aumento do número de parceiros. $\mathrm{O}$ odds ratio com um único parceiro foi 2,64 (IC95\%: 1,42-4,92).

O maior tempo de vida sexual apresenta associação significante somente entre as moças. 
Análise de regressão logística multinomial, ajustada, das variáveis associadas ao uso de preservativo pelos jovens de 18 a 24 anos, na primeira relação sexual, em Porto Alegre, Rio de Janeiro e Salvador, Brasil, $2002(\mathrm{~N}=4.019)$.

\begin{tabular}{|c|c|c|c|c|}
\hline \multirow[t]{2}{*}{ Nível* $^{*}$} & \multicolumn{2}{|c|}{ Homens } & \multicolumn{2}{|c|}{ Mulheres } \\
\hline & $\begin{array}{l}\text { Preservativo } \\
\text { OR (IC95\%) }\end{array}$ & $\begin{array}{l}\text { Outro método } \\
\text { OR (IC95\%) }\end{array}$ & $\begin{array}{l}\text { Preservativo } \\
\text { OR (IC95\%) }\end{array}$ & $\begin{array}{l}\text { Outro método } \\
\text { OR (IC95\%) }\end{array}$ \\
\hline \multicolumn{5}{|l|}{1} \\
\hline \multicolumn{5}{|l|}{ Idade na entrevista (anos) } \\
\hline Até 19 & $1,69(1,19-2,40)$ & $0,95(0,42-2,13)$ & $1,69(1,19-2,38)$ & $0,61(0,33-1,15)$ \\
\hline$>19$ & 1,00 & 1,00 & 1,00 & 1,00 \\
\hline \multicolumn{5}{|l|}{ Cor } \\
\hline Branca & $1,21(0,84-1,76)$ & $0,90(0,46-1,74)$ & $1,83(1,37-2,43)$ & $1,53(0,88-2,65)$ \\
\hline Negro/Pardo & 1,00 & 1,00 & 1,00 & 1,00 \\
\hline \multicolumn{5}{|l|}{ Escolaridade da mãe (anos) } \\
\hline Nenhuma & $1,00^{\star \star}$ & 1,00 & $1,00^{\star \star}$ & 1,00 \\
\hline Até 7 & $1,43(0,76-2,71)$ & $0,48(0,21-1,09)$ & $1,28(0,67-2,47)$ & $0,45(0,17-1,20)$ \\
\hline $8-10$ & $2,62(1,27-5,40)$ & $0,44(0,11-1,72)$ & $1,71(0,81-3,61)$ & $0,70(0,27-1,78)$ \\
\hline $11 \mathrm{ou}+$ & $2,63(1,36-5,07)$ & $0,35(0,11-1,11)$ & $2,42(1,24-4,73)$ & \\
\hline \multicolumn{5}{|l|}{2} \\
\hline \multicolumn{5}{|l|}{ Escolaridade do jovem (anos) } \\
\hline Até 7 & 1,00 & 1,00 & $1,00 * \star$ & 1,00 \\
\hline $8-10$ & $1,62(1,09-2,41)$ & $1,38(0,75-2,53)$ & $1,73(1,12-2,67)$ & $1,84(0,93-3,63)$ \\
\hline 11 ou + & $1,34(0,89-2,01)$ & $0,69(0,30-1,56)$ & $2,53(1,60-3,98)$ & $5,04(2,66-9,56)$ \\
\hline \multicolumn{5}{|l|}{ Segue religião } \\
\hline Sim & $1,40(1,06-1,85)$ & $1,70(0,80-3,61)$ & $1,30(0,99-1,71)$ & $1,23(0,79-1,93)$ \\
\hline Não & 1,00 & 1,00 & 1,00 & 1,00 \\
\hline \multicolumn{5}{|l|}{3} \\
\hline \multicolumn{5}{|l|}{ Primeiras informações sobre contracepção com mãe } \\
\hline Sim & $1,49(1,00-2,23)$ & $1,60(0,55-4,61)$ & $0,97(0,70-1,35)$ & $0,75(0,48-1,18)$ \\
\hline Não & 1,00 & 1,00 & 1,00 & 1,00 \\
\hline \multicolumn{5}{|l|}{ Primeiras informações sobre contracepção com pai } \\
\hline $\operatorname{Sim}$ & $1,55(1,08-2,23)$ & $1,46(0,41-5,23)$ & $0,97(0,47-2,04)$ & $0,47(0,16-1,36)$ \\
\hline Não & 1,00 & 1,00 & 1,00 & 1,00 \\
\hline \multicolumn{5}{|l|}{ Parceiro da primeira relação sexual } \\
\hline Pessoa com quem ficou/Garoto(a) de programa & 1,00 & 1,00 & 1,00 & 1,00 \\
\hline Namorado(a)/Marido/Esposa/Companheiro(a) & $0,76(0,54-1,07)$ & $1,35(0,60-3,01)$ & $0,83(0,48-1,44)$ & $1,43(0,46-4,42)$ \\
\hline \multicolumn{5}{|l|}{ Idade na primeira relação sexual (anos) } \\
\hline Precoce (rapazes: até 14; moças: até 15) & $1,00 * \star \star$ & 1,00 & $1,00 * \star \star$ & 1,00 \\
\hline Média (rapazes: 15-16; moças: 16-17) & $3,48(2,31-5,22)$ & $2,41(0,84-6,88)$ & $1,38(0,92-1,95)$ & $1,34(0,67-2,69)$ \\
\hline Tardia (rapazes: 17-24; moças: 18-24) & $3,96(2,57-6,10)$ & $3,46(1,65-7,33)$ & $1,98(1,24-3,17)$ & $2,30(1,15-4,60)$ \\
\hline \multicolumn{5}{|l|}{ Diferença etária entre parceiros } \\
\hline Parceria mais jovem ( 2 anos e + ) & $1,00 * * \star$ & 1,00 & 1,00 & 1,00 \\
\hline Mesma idade (entre 1 ano a - ou + ) & $2,60(1,30-5,20)$ & $1,06(0,38-2,94)$ & $0,41(0,11-1,59)$ & $0,25(0,04-1,57)$ \\
\hline Parceria mais velha (entre $2-4$ anos a + ) & $3,14(1,40-7,06)$ & $1,57(0,54-4,55)$ & $0,48(0,13-1,79)$ & $0,37(0,06-2,38)$ \\
\hline Parceria muito mais velha $(5$ anos $\mathrm{e}+$ ) & $4,44(1,58-2,48)$ & $1,55(0,49-4,94)$ & $0,68(0,18-2,58)$ & $0,49(0,08-2,78)$ \\
\hline \multicolumn{5}{|l|}{$\begin{array}{l}\text { Conversaram sobre contracepção antes } \\
\text { da primeira relação sexual }\end{array}$} \\
\hline Sim & $4,91(3,38-7,14)$ & $5,03(2,10-12,02)$ & $4,36(3,12-6,09)$ & $8,00(4,19-15,28)$ \\
\hline Não & 1,00 & 1,00 & 1,00 & 1,00 \\
\hline
\end{tabular}

* Todas as variáveis estão controladas para as demais do mesmo nível e para as dos níveis superiores. A categoria de referência do desfecho é nenhum método;

** As variáveis com nível de significância entre $5 \%$ e $20 \%$ foram mantidas no modelo para controle de confusão;

*** $\mathrm{O}$ teste de Wald para tendência apresentou valor $\mathrm{p}<0,05$. 
Tabela 5

Análise de regressão logística multinomial, ajustada, das variáveis associadas ao uso de preservativo pelos jovens de 18 a 24 anos, na última relação sexual, em Porto Alegre, Rio de Janeiro e Salvador, Brasil, 2002 ( $N=3.836$ ).

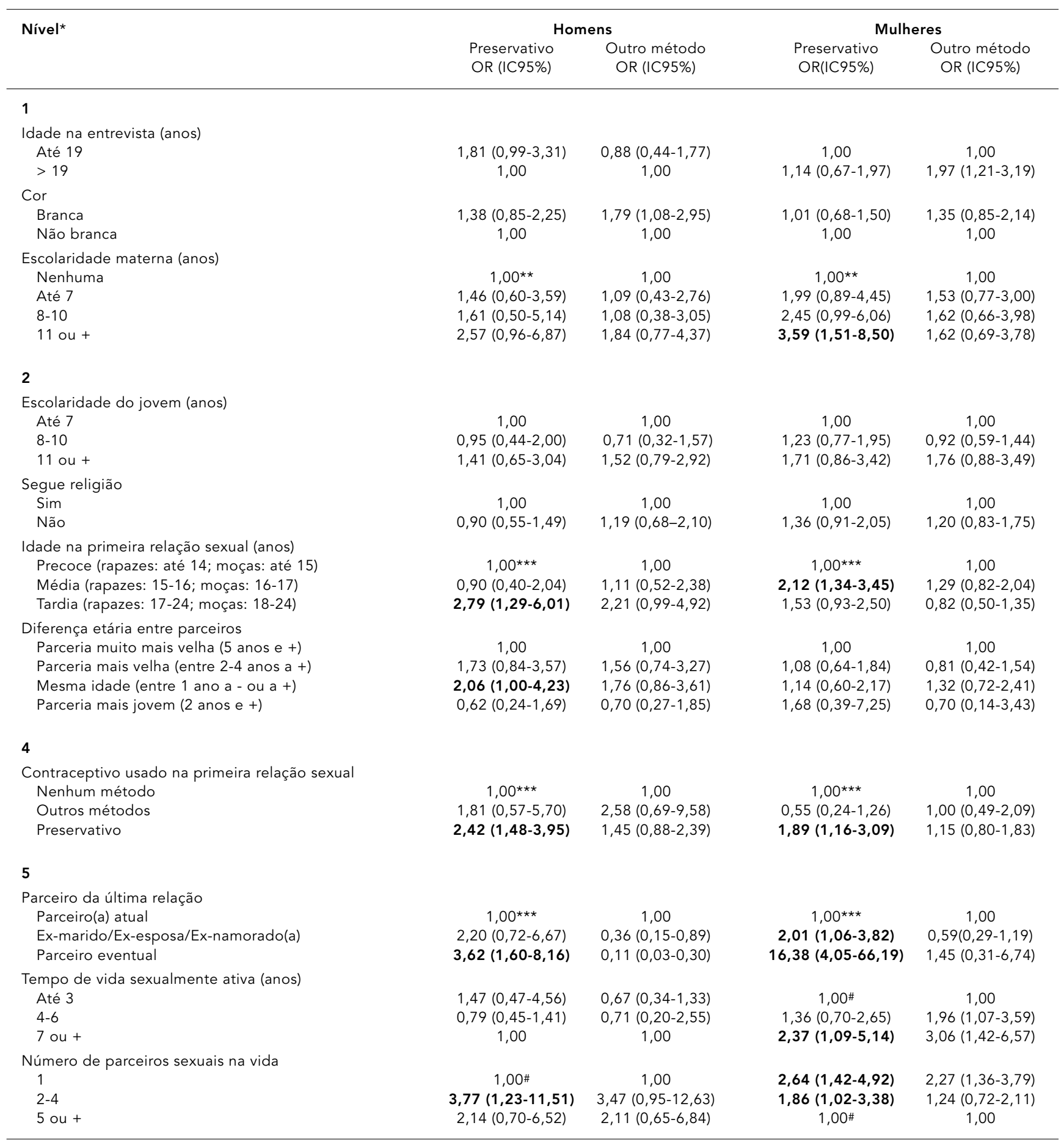

* Todas as variáveis estão controladas para as demais do mesmo nível e para as dos níveis superiores. A categoria de referência do desfecho é nenhum método. As variáveis do 3o nível, primeiras informações sobre métodos com a mãe e com o pai, quem era o parceiro da 1a relação sexual e conversaram sobre contracepção antes da 1a relação sexual foram retiradas do modelo (valor $\mathrm{p}>0$,2). Idem com tipos de trajetórias do 5o nível. Estes dados não foram apresentados na tabela acima;

** As variáveis com nível de significância entre $5 \%$ e $20 \%$ foram mantidas no modelo para controle de confusão;

$\star \star \star ~ O$ teste de Wald para tendência apresentou valor $p<0,05$;

\# O teste de Wald para heterogeneidade apresentou valor $p<0,05$. 
Aquelas que tinham sete anos ou mais de vida sexual apresentaram OR =2,37 (IC95\%: 1,09$5,14)$, quando comparadas com aquelas que tinham até três anos de vida sexual. Entre os rapazes a variável perdeu a associação.

A idade do jovem por ocasião da entrevista, até 19 anos, comparando com os mais velhos não apresentou associação na última relação. Já o comportamento da variável foi diferente na iniciação sexual, quando o uso de preservativo apresentou OR = 1,69 (IC95\%: 1,19-2,40), significativamente associado para ambos os sexos, entre os mais jovens.

A variável diferença de idade entre parceiros apresentou associação somente para os rapazes, tanto na última relação sexual como na iniciação. Porém, a influência da variável foi mais marcada na iniciação, principalmente com as parceiras mais velhas e muito mais velhas, apresentando OR = 3,14 (IC95\%: 1,40-7,06) e OR = 4,44 (IC95\%: 1,58-2,48), respectivamente.

Na iniciação sexual (Tabela 4), além das variáveis já citadas na comparação com a última relação, escolaridade do jovem elevada e ter conversado sobre métodos anticoncepcionais antes da primeira relação também apresentaram associação com o uso de preservativo, para ambos os sexos. Cor da pele branca mostrou associação somente para as moças. Seguir religião e receber as primeiras informações sobre métodos com o pai mostraram-se associados somente para os rapazes.

\section{Discussão}

O uso do preservativo é determinado por fatores não só de ordem sócio-cultural, como também de ordem situacional e individual. Quando analisamos os fatores que apareceram associados ao uso do preservativo nos dois eventos estudados, observamos que o pertencimento social (cuja proxy utilizada é a escolaridade da mãe) e a idade da iniciação sexual exercem forte influência para ambos os sexos. Neste estudo, o pertencimento social aparece como mais decisivo no comportamento dos jovens do que outras variáveis de cunho mais individual, como é o caso da escolaridade do entrevistado. Na iniciação sexual, a escolaridade da mãe está associada ao uso de preservativo para ambos os sexos, chegando a superar a do entrevistado como fator determinante, como é o caso entre os rapazes. Já na última relação sexual, a escolaridade do entrevistado não se associa ao uso do preservativo, enquanto a escolaridade da mãe permanece associada entre as moças e, entre os rapazes, observa-se uma tendência de aumento do uso do preservativo diretamente proporcional ao aumento da escolaridade da mãe. Na literatura, o uso de preservativo na iniciação sexual também é mais elevado na população mais instruída e nos estratos sócio-econômicos mais altos 8,12,13.

A iniciação sexual mais tardia foi de extrema importância na determinação do uso de preservativo nos dois eventos e para ambos os sexos. O efeito foi mais marcado na primeira relação e entre os rapazes. Béria 5 em um estudo com adolescentes encontrou que o risco de não usar preservativo na última relação se reduzia em $18 \%$ para cada ano a mais de idade na primeira relação sexual, para ambos os sexos. Longo 4, analisando dados do Demographic and Health Surveys de 1996, apesar de não avaliar especificamente o uso de preservativo, mostrou que as mulheres com iniciação sexual mais tardia utilizaram mais métodos anticoncepcionais na iniciação que aquelas que se iniciaram até os 15 anos. Almeida et al. 2, ao estudarem jovens de Salvador, encontraram associação positiva com o uso de contraceptivos entre os rapazes com iniciação sexual mais tardia, de 15 a 19 anos. Narring et al. 14 encontraram que jovens suíços, de ambos os sexos, usam menos preservativo quando a iniciação sexual é abaixo dos 15 anos de idade.

Um outro achado interessante do estudo é o de que o uso do preservativo na iniciação sexual aumenta a probabilidade de uso na última relação. Este dado mostra que os jovens que usam preservativo na iniciação tendem a manter esta prática, no decorrer de sua vida sexual, o que reforça a necessidade de orientação continuada para o estímulo ao uso do preservativo. A associação foi para ambos os sexos e também aparece descrita na literatura 4 .

Os dados analisados sobre a prevalência do uso do preservativo entre os jovens corroboram a literatura sobre o tema, que aponta para o aumento do uso em relação a alguns anos atrás. Mas mesmo com este aumento nos últimos anos, deve-se observar que o uso desse método não se mantém nos mesmos percentuais quando comparamos dois eventos, no caso a primeira e a última relação sexual. O uso do preservativo cai significativamente na última relação sexual, particularmente entre as mulheres, que também o utilizam num percentual menor que os homens na iniciação 1,12,15,16.

Essa diferença nas escolhas masculinas e femininas, que também é apontada por outros estudos 2,3,5,7,8,15,16,17, sinaliza para a diferença de gênero no que concerne às concepções e preocupações vinculadas aos relacionamentos afetivo-sexuais, bem como às experiências di- 
versas neste campo. Ao longo de sua trajetória, pouco mais de um terço das mulheres entrevistadas teve apenas um parceiro sexual, ao passo que as trajetórias masculinas se caracterizam por uma maior variedade de parcerias. Para os homens, a diversidade de parceiras implica o maior uso do preservativo 2, enquanto para as mulheres observa-se uma relação inversa. $\mathrm{Ou}$ seja, para estas, a diversidade de parceiros, talvez por as colocar num status social "questionável” (vinculado às concepções de promiscuidade sexual), aparece relacionada à diminuição do uso do preservativo. É interessante notar aqui que essas mulheres, possivelmente, encontram maior dificuldade em negociar com os parceiros não apenas o uso do preservativo, mas também o estatuto do próprio relacionamento 16 .

Além do número de parceiros, a mudança do estatuto do relacionamento, ou seja, de eventual para estável, implica ainda a diminuição do uso de preservativo e o conseqüente aumento do uso de outros métodos de contracepção, como a pílula anticoncepcional. É assim que o uso do preservativo é maior, na última relação sexual, tanto para homens quanto para mulheres, quando esta se dá com um parceiro eventual 8,13,14,15. Nos relacionamentos considerados estáveis, a prioridade deixa de ser a proteção das infecções de transmissão sexual e passa ser a prevenção da gravidez.

Um outro elemento que contribui para essa mudança de método é a diferença etária na composição dos casais, visto que as moças se relacionam mais freqüentemente com parceiros mais velhos que, por sua vez, tendem a usar menos preservativo. Nesse sentido, deve-se salientar que a diferença etária entre os parceiros apresentou associação somente entre os rapazes, em particular no contexto da iniciação sexual, em que aqueles que se relacionaram com mulheres mais velhas (entre dois a quatro anos a mais e cinco anos e mais) foram os que mais utilizaram preservativo 14 . As mulheres mais velhas que se relacionam com homens mais jovens parecem dispor de maior poder de negociar em relação ao uso da camisinha, ao passo que as mais jovens encontram-se numa situação de maior vulnerabilidade 7,18. Um dado que vem corroborar essa hipótese é o de que o maior tempo de vida sexual aparece associado ao uso do preservativo apenas entre as mulheres, ou seja, o poder de negociação do uso do preservativo é adquirido, pelas mulheres, com a idade e com a experiência.

O uso de preservativos depende mais de variáveis situacionais e temporais que o uso de outros métodos. A contracepção é uma norma mais internalizada nas mulheres, enquanto que o uso de preservativos requer delas habilidades de negociação.

É importante salientar que, se a idade é uma das variáveis associadas ao uso do preservativo, as variáveis contextuais, tais como a diferença etária do casal e o evento analisado, tornam essa associação complexa. A tendência encontrada em vários estudos de os mais jovens usarem mais preservativos que os mais velhos $1,3,4,7,8$, $13,14,19$ não se apresenta da mesma forma quando analisamos a primeira e a última relação sexual, nem mesmo quando olhamos para as diferenças entre os sexos. Os mais jovens, na ocasião da pesquisa, com idade até 19 anos, comparados com os mais velhos, apresentaram associação limítrofe com o uso de preservativo na última relação e somente para os rapazes, diferentemente do que acontece na iniciação, quando o uso esteve significativamente associado entre os mais jovens de ambos os sexos.

O maior uso de preservativos entre os mais jovens e na primeira relação sexual deve-se, por um lado, ao advento da AIDS e ao sucesso de suas campanhas de prevenção, uma vez que a geração mais nova já nasceu sob o impacto da epidemia, parecendo ser mais permeável à adoção do uso do preservativo que os mais velhos, que se iniciaram sexualmente sem essa ameaça 7,17. Por outro lado, deve-se a um maior investimento no planejamento da primeira relação sexual 14,20 e ao fato de que, entre os rapazes, muitas vezes, a primeira relação se dá com uma parceira considerada como eventual e, portanto, desconhecida 14,21 .

No que concerne à relação entre uso do preservativo e informações sobre proteção/contracepção, cabe salientar a importância da família, particularmente no universo masculino. Mesmo tendo sido pequeno o percentual de rapazes que referiram ter recebido do pai as primeiras informações sobre métodos anticoncepcionais, observa-se sua influência na adoção do preservativo no contexto da primeira relação sexual. Ter recebido da mãe as primeiras informações apresentou associação limítrofe somente com rapazes. Isso reforça a importância do pai como fonte de orientação e esclarecimento, pois, como sugere a literatura, pais mais democráticos e que promovem o diálogo com os filhos são mais capazes de protegê-los e orientá-los 22.

O diálogo sobre contracepção/proteção entre os parceiros também interfere positivamente no uso do preservativo, como indica o dado de que as moças e os rapazes que conversaram sobre métodos antes da iniciação utilizaram mais preservativo nessa relação. Esse dado si- 
naliza para a importância de as políticas de prevenção de DST/AIDS e de educação sexual enfocarem o caráter relacional do uso do preservativo e não tomá-lo apenas sob a ótica da responsabilidade individual 5,23,24.

Uma possível limitação deste estudo está relacionada ao viés de memória. Fizeram-se perguntas sobre situações que aconteceram, para alguns dos jovens, há vários anos, entretanto, como o estudo indagou sobre eventos marcantes da vida deles (como a iniciação sexual) e como priorizou igualmente eventos recentes (como a última relação sexual), esperase ter minimizado esse problema. Nesse sentido, deve-se lembrar que se trabalhou com base nas declarações dos jovens, existindo a possibilidade de os relatos refletirem comportamen-

\section{Resumo}

O uso de preservativos pelos jovens aumentou, o que não significa que sejam utilizados em todas as relações sexuais; além disso, seu uso varia durante a trajetória afetivo-sexual. Estudou-se a prevalência e fatores associados ao uso de preservativo na iniciação sexual e na última relação sexual, para moças e rapazes de 18-24 anos. Os dados são da Pesquisa GRAVAD, um estudo transversal com amostra probabilística estratificada, através de entrevistas domiciliares, em três capitais brasileiras. Na análise, utilizou-se regressão logística multinomial seguindo modelo hierarquizado. A prevalência de uso de preservativo na iniciação, dentre os usuários de métodos contraceptivos, foi 80,7\% pelas moças e 88,6\% pelos rapazes. Este uso cai para 38,8\% e 56\%, respectivamente, na última relação. Nos dois eventos, o uso de preservativo esteve associado, para ambos os sexos, ao pertencimento social e à idade da iniciação. A utilização do preservativo na iniciação determina o uso na última relação $(O R=2,42$ para os rapazes e 1,89 para as moças). O maior uso de preservativo entre os jovens não implica uso continuado. As moças utilizaram menos preservativo, comparadas aos rapazes, nos eventos estudados.

Adolescente; Sexualidade; Preservativos; Identidade de Gênero tos idealizados e não, necessariamente, eventos concretos 19. Além de consistência interna das informações para ambos os sexos, os achados são consistentes com outros estudos de base populacional, sobre os mesmos eventos e que tiveram um tempo de recordatório menor ou semelhante a este 2,5,19.

Por fim, cabe salientar que o maior uso de preservativo entre os jovens não implica um uso continuado. Daí a importância de estudos que avaliem a consistência do uso de preservativo e que possibilitem definir estratégias para aumentar a sua utilização no decorrer da vida. Ênfase especial deve ser dada para as mulheres, que se encontram em situação de desvantagem, comparadas aos homens, tanto na primeira como na última relação.

\section{Colaboradores}

A. M. F. B. Teixeira participou da revisão bibliográfica, limpeza dos dados, criação de variáveis, análise estatística e redação do artigo. D. R. Knauth participou do planejamento e execução da pesquisa, da revisão bibliográfica, limpeza dos dados, criação de variáveis, análise estatística e revisão da redação do artigo. J. M. G. Fachel participou do planejamento e execução da pesquisa, da revisão bibliográfica, criação de variáveis, orientação da análise estatística e revisão da redação do artigo. A. F. Leal participou do planejamento e execução da pesquisa, da coleta e limpeza dos dados, criação de variáveis e revisão do artigo.

\section{Agradecimentos}

A investigação Gravidez na Adolescência: Estudo Multicêntrico sobre Jovens, Sexualidade e Reprodução no Brasil (Pesquisa GRAVAD) foi elaborada por Maria Luiza Heilborn (Instituto de Medicina Social, Universidade do Estado do Rio de Janeiro - IMS/UERJ), Michel Bozon (Institute National d'Études Démographiques - INED, França), Estela M. L. Aquino (Programa Integrado de Pesquisa e Cooperação Técnica em Gênero e Saúde/Universidade Federal da Bahia MUSA/UFBA) e Daniela Knauth (Núcleo de Antropologia do Corpo e Saúde/Universidade Federal do Rio Grande do Sul - NUPACS/UFRGS). O estudo foi realizado por três centros: Programa em Gênero, Sexualidade e Saúde (IMS/UERJ), MUSA/UFBA e NUPACS/ UFRGS. Os principais resultados do inquérito encontram-se publicados no livro O Aprendizado da Sexualidade: Reprodução e Trajetórias Sociais de Jovens Brasileiros (Rio de Janeiro: Garamond; 2006), onde podem ser obtidas informações sobre a composição detalhada da equipe de pesquisadores. Agradecemos também à Fundação Ford, ao programa de bolsas do Conselho Nacional de Desenvolvimento Científico e Tecnológico e à Coordenação de Aperfeiçoamento de Pessoal de Nível Superior. 


\section{Referências}

1. Pinho MDG, Berquó E, Lopes F, Oliveira KA, Lima LCA, Pereira N. Juventudes, raça e vulnerabilidades. Revista Brasileira de Estudos Populacionais 2002; 19:277-94.

2. Almeida MC, Aquino EM, Gaffikin L, Magnani RJ. Contraceptive use among adolescents at public schools in Brazil. Rev Saúde Pública 2003; 37:56675.

3. Camarano AA. Fecundidade e anticoncepção da população jovem. In: Berquó E, organizador. Jovens acontecendo na trilha das políticas públicas. Brasília: Comissão Nacional de População e Desenvolvimento; 1998. p. 109-33.

4. Longo LAFB. Juventude e contracepção: um estudo dos fatores que influenciam o comportamento contraceptivo das jovens brasileiras de 15 a 24 anos. Revista Brasileira de Estudos Populacionais 2002; 19:229-47.

5. Béria J. Ficar, transar. A sexualidade do adolescente em tempos de AIDS. Porto Alegre: Tomo Editorial; 1998.

6. Castilho EA, Szwarcwald CL. Mais uma pedra no meio do caminho dos jovens brasileiros: a AIDS. In: Berquó E, organizador. Jovens acontecendo na trilha das políticas públicas. Brasília: Comissão Nacional de População e Desenvolvimento; 1998. p. 197-207.

7. Castro MG, Abramovay M, Silva LB. Juventudes e sexualidade. Brasília: UNESCO Brasil; 2004.

8. Centro Brasileiro de Análise e Planejamento. Comportamento sexual da população brasileira e percepções do HIV/AIDS. 1ạ Ed. Brasília: Ministério da Saúde; 2000.

9. Aquino EM, Heilborn ML, Barbosa RM, Berquó E. Gênero, sexualidade e saúde: a constituição de um novo campo na saúde coletiva [Editorial]. Cad Saúde Pública 2003; 19 Suppl 2:S198-9.

10. Victora CG, Huttly SR, Fuchs SC, Olinto MT. The role of conceptual frameworks in epidemiological analysis: a hierarchical approach. Int J Epidemiol 1997; 26:224-7.

11. Maldonado G, Greeland S. Simulation study of confounder-selection strategies. Am J Epidemiol 1993; 138:923-36.

12. Caballero-Hoyos R, Villasenor Sierra A. Socioeconomic strata as a predictor factor for constant condom use among adolescents. Rev Saúde Pública 2001; 35:531-8.

13. Paiva V, Venturi G, França Jr. I, Lopes F. Uso de preservativos. Brasília: Ministério da Saúde; 2003.
14. Narring F, Wydller H, Michaud P. First sexual intercourse and contraception: a cross-sectional survey on the sexuality of 16 - 20-year-olds in Switzerland. Schweiz Med Wochenschr 2000; 130:1389-98.

15. Pirotta KCM, Schor N. Juventude e saúde reprodutiva: valores e condutas relacionados com a contracepção entre universitários. In: XIII Encontro da Associação Brasileira de Estudos Populacionais; 2002. http://www.abep.nepo.unicamp. br/docs/anais/pdf/2002/Com_JUV_ST7_Pirotta_ texto.pdf.

16. Richter DL, Valois RF, McKeown RE, Vincent ML. Correlates of condom use and number of sexual partners among high school adolescents. J Sch Health 1993; 63:91-6.

17. Arredondo A, Goldstein E, Oliveira MP, Bozon M, Giraud M, Mesich A, et al. Estudio nacional de comportamiento sexual: primeros analisis. Santiago de Chile: Comisión Nacional el Sida, Ministerio de Salud; 2000.

18. Barbosa RM. Negociação sexual ou sexo negociado? Poder, gênero e sexualidade em tempos de AIDS. In: Barbosa RM, Parker R, organizadores. Sexualidades pelo avesso: direitos, identidades e poder. Rio de Janeiro: Instituto de Medicina Social, Universidade do Estado do Rio de Janeiro/ São Paulo: Editora 34; 1999. p. 73-88.

19. Silveira MF, Santos IS, Béria JU, Horta BL, Tomasi E, Victora CG. Factors associated to condom use among women of an urban center in southern Brazil. Cad Saúde Pública 2005; 21:1557-64.

20. Bozon M, Heilborn ML. As carícias e as palavras: iniciação sexual no Rio de Janeiro e em Paris. Novos Estudos CEBRAP 2001; 59:111-35.

21. Guimarães CD. Mas eu conheço ele: um método de prevenção do HIV/AIDS. In: Parker R, Galvão J, organizadores. Quebrando o silêncio. Mulheres e AIDS no Brasil. Rio de Janeiro: Relume-Dumará; 1996. p. 169-79.

22. Boruchovitch E. Factors associated with the nonutilization of contraceptives in adolescence. Rev Saúde Pública 1992; 26:437-43.

23. Paiva V. Fazendo arte com camisinha: a história de um projeto de prevenção de AIDS para jovens. São Paulo: Summus; 2000.

24. Parker R. Na contramão da AIDS: sexualidade intervenção e política. Rio de Janeiro: Associação Brasileira Interdisciplinar de AIDS; 2000.

Recebido em 20/Abr/2005

Versão final reapresentada em 19/Jul/2005

Aprovado em 04/Ago/2005 